\title{
Mitral Valve - Innocent Bystander or Master of its Own Destiny?
}

\author{
Pradeep Narayan ${ }^{1}$ and Gianni Angelini² \\ ${ }^{1}$ Rabindranath Tagore International Institute of Cardiac Sciences \\ ${ }^{2}$ Bristol University
}

October 5, 2020

\begin{abstract}
It has been long believed that ischemic mitral regurgitation is secondary to left ventricular remodelling and the mitral per se remains unaffected. This proviso has recently been challenged and the mitral valve has been described as a structure that responds and adapts to challenges and attempts to correct the mitral regurgitation. The response of mitral valves in this setting can be incomplete or can even be mal-adapted. The ability of the mitral valve to respond in this manner has been described as "mitral plasticity". Endothelial to Mesenchymal transition and Valvular Interstitial Cells are key to this mitral plasticity and function through a complex array of signalling pathways. Identification and manipulation of these pathways may provide a possibility to correct the incomplete or mal-adapted mitral valve responses. Surgical treatment can also be tailored based on whether the valve has maladapted or has undergone incomplete adaptation.
\end{abstract}

\section{Mitral Valve - Innocent Bystander or Master of its Own Destiny?}

Pradeep Narayan FRCS[CTh]*, Gianni D Angelini FRCS^^

*NH Rabindranath Tagore International Institute of Cardiac Sciences, Kolkata, India

Short Title: Role of Mitral Plasticity

Word Count : 1406

\section{Corresponding Author:}

Prof GD Angelini, MD,MCh,FRCS, FMedSci

British Heart Foundation Professor of Cardiac Surgery,

Bristol Heart Institute Bristol Royal Infirmary,

Upper Maudlin Street BS2 8HW

Bristol, UK

G.D.Angelini@bristol.ac.uk

Conflict of Interest: none

Funding: This work was supported by the British Heart Foundation and the NIHR Biomedical Research Centre at University Hospitals Bristol and Weston NHS Foundation Trust and the University of Bristol.

Key words: Mitral Plasticity, Endothelial-Mesenchymal Transition, Valvular Interstitial Cells.

The pathophysiology of functional mitral regurgitation has traditionally been described as being controlled by changes occurring in the left ventricle. It was believed that changes in the left ventricle led to changes in the orientation of the different components of the Mitral Valve apparatus- leaflets, chorda tendinae and the 
papillary muscles. If the orientation of the mitral valve apparatus was not satisfactory it resulted in mitral regurgitation. Thus, the MV was portrayed as an innocent bystander whose fate and functioning were in the hands of the left ventricle.

However, in this excellent manuscript by Calafiore et $\mathrm{al}^{1}$ this premise of the MV being an inert bystander has been challenged. The authors have suggested that the MV is not an inert structure but one that responds to changes and challenges in its surroundings. Depending on its ability to respond to these changes adequately or inadequately determines if the MV stays competent or becomes incompetent. Its ability to respond to these changes is determined by what the authors call- "Mitral Plasticity". The authors propose that Mitral Plasticity is an equally important determinant in the pathophysiology of MR and not just the left ventricle as previously believed. The authors also suggest that interventions for MR, pharmacological or surgical, should therefore focus on modifying Mitral Plasticity for a successful outcome.

The concept of Mitral valve plasticity is quite simple. It is the ability of the Mitral valve to adapt to stresses imposed by the failing left ventricle or to the turbulence created by a regurgitant jet of $\mathrm{MR}^{2,3}$ In response to these stresses the mitral valve leaflet can increase its surface area thereby increasing the area of coaptation resulting in reduction of MR. This does not happen in all cases and in some there is incomplete adaptation or even mal-adaptation. The mal-adaptation can lead to undesirable changes like thickening and fibrosis of leaflets or chordae. While incomplete adaptation leads to persistence of MR, mal-adaptation may lead to even exaggeration of MR.

However, Plasticity of Mitral valves is not a macroscopic phenomenon, but a cellular concept whereby one type of cell can change into another type depending on the need and still retain its ability to return to its original type- otherwise referred as being able to "assume different cellular phenotypes." ${ }^{4}$ Plasticity of cells is a normal developmental phenomenon and the concepts of adaptation and mal-adaptation can be seen during wound repair and in cancer cells respectively. This plasticity happens by conversion of epithelial cells to mesenchymal cell, also known as Epithelial to Mesenchymal Transition (EMT). The term transition denotes its reversibility as opposed to the term transformation which is irreversible. The same phenomenon when seen in endothelial cells is called Endothelial to Mesenchymal Transition (EndMT). Transition to mesenchymal cells leads to formation of myofibroblasts as well as smooth muscle cells.

This concept of EndMT is important because in the mitral valve the leaflets are covered by endothelial cells on atrial as well as ventricular sides. ${ }^{5}$ Therefore EndMT is central to "plasticity" of mitral valves. In fact, the role of EndMT is paramount in several other situations as well. Saphenous vein bypass graft failure is the prime example of EndMT. Exposed to the stress of higher arterial blood pressure after CABG, the venous endothelium undergoes a mal-adaptive EndMT that results in smooth muscle cell hyperplasia and eventually graft failure. ${ }^{6}$ Similarly EndMT affecting the ventricular endothelium results in fibroelastosis. Coronary atherosclerosis and cardiac transplant vasculopathy are other pathologies that have been suggested to be influenced by EndMT. ${ }^{6,7}$ Studies have reported that roughly $1 \%$ of adult endothelial cells are capable of EndMT. 8

The second important concept described by the authors is the role of Valvular Interstitial Cells (VICs) in disease process. VICs are found in all the layers of the valve and are responsible for the structural integrity of the valve. They are normally quiescent but in disease states these cells get activated. Simplistically speaking, mechanical stress and inflammatory changes lead to EndMT which leads to activation and generation of VICs. ${ }^{9}$ These VICs then regulate the repair and remodelling through a complex interplay of various mediators and pathways described by the authors. ${ }^{6}$

While the concept of Mitral Plasticity and how it may affect the degree of MR is fascinating the more clinically relevant aspect is identifying factors that can potentially influence plasticity. This will provide clinicians the required knowledge to predict the degree of secondary $\mathrm{MR}$ in a particular case. Even more importantly, from a therapeutic point of view it may well be possible to intervene either pharmacologically or surgically to complete or correct the adaptation. From a clinical perspective it is therefore important to identify factors that initiate and influence the process of Mitral Plasticity. The common clinical scenarios which 
initiate Mitral Plasticity are mitral regurgitation, myocardial infarctions and LV dilatation. ${ }^{2,3,10}$ Following an infarction there can be asymmetric LV remodelling that leads to tenting. ${ }^{11}$ LV dilatation displaces the papillary muscles outwards also leading to tethering of the mitral valve. ${ }^{3}$ The regurgitant jet of MR also stresses the leaflet on the atrial side. The mitral valve responds to these stimuli and increases its surface area. ${ }^{12}$ This adaptive mechanism has been confirmed in animal models and other studies. ${ }^{2,10,12}$

While the accepted theory is that Mitral Plasticity is an adaptive response to LV remodelling it has also been noted that even with similar degree of LV remodelling there can be dissimilar degrees of Mitral Plasticity. ${ }^{3}$ This suggests that LV remodelling may be responsible for initiating the Mitral Plasticity but there must be factors which influence the degree of adaptation. Some of the clinically relevant factors that have been recognized to influence the process of plasticity include duration of diabetic control, hemoglobin levels and smoking. ${ }^{3}$ These factors provide part of the explanation why there is variability in this adaptive response.

This and knowledge of the factors that influence plasticity provide an exciting opportunity to manipulate and modulate the process of Mitral Plasticity. The management can thus be tailored to the response. Pharmacological measures, for instance, can be taken to improve the ability of the mitral valve to adapt adequately. Type of surgical repair can be tailored depending on whether it is incomplete adaptation or mal-adaptation that is the pathology behind the persistent MR.

In fact, work in this direction has already begun. It has been reported that Angiotensin II receptors are found on the valvular endothelial cells of the mitral valve which are stimulated by Angiotensin II to initiate the process of EndMT. This response can be effectively blocked by using Angiotensin II receptor blockers. ${ }^{13}$ This property therefore has the potential to prevent excessive EndMT that may result in fibrosis of the valve leaflets. However, as elegantly described by the authors, EndMT is controlled by complex signalling networks, and not all of them are completely understood. ${ }^{14}$ Researchers working on tissue engineered valves have taken cue from the fact that EMT is the first step for the endocardial cushions to become a mature valve leaflet. As a result cell types which can induce EMT are considered to be a great option for the valve scaffolds. ${ }^{15}$ The principle of leaflet augmentation in mitral valve repair is nothing but completing the process of incomplete adaptation. The thickened retracted chords can be seen as a mal-adaptation response and cutting the second order chords have been used to treat the process of mal-adaptation with satisfactory outcome. $^{16}$

While the concept brought in by the authors is novel, even revolutionary, realistically speaking we are still in the nascent stage of this concept of "Mitral Plasticity" both from clinical as well as cellular perspectives. In the current phase effort must be directed towards better understanding of the process of EndMT and the interplay between EndMT and VICs. Confirmatory mapping of the inhibitory and activating signals for EndMT must be done before models to manipulate EndMT and Mitral Plasticity can be reliably drawn. Clinically, further studies would be required to identify co-morbid conditions that influence "Mitral Plasticity" and only then can we think of intervening pharmacologically to achieve desired outcomes. Surgical interventions based on the concepts of Mitral Plasticity have to be done in larger studies and only then convincing evidence can be gathered. It is undoubtedly going to be a long hard road but one that is worth travelling and the authors must be once again congratulated for taking the first few steps.

\section{References}

1. Calafiore A, Totaro A, Testa N, Sacra C, Castellano G, Guarracini S, et al. THE SECRET LIFE OF MITRAL VALVE [Internet]. Preprints; 2020 Sep [cited 2020 Oct 3]. J Card Surg. 2020 xxxxxxxx

2. Dal-Bianco Jacob P., Aikawa Elena, Bischoff Joyce, Guerrero J. Luis, Handschumacher Mark D., Sullivan Suzanne, et al. Active Adaptation of the Tethered Mitral Valve. Circulation. 2009 Jul 28;120(4):334-42.

3. Ávila-Vanzzini N, Michelena HI, Fritche Salazar JF, Herrera-Bello H, Siu Moguel S, Rodríguez Ocampo RR, et al. Clinical and echocardiographic factors associated with mitral plasticity in patients with chronic inferior myocardial infarction. Eur Heart J - Cardiovasc Imaging. 2018 May 1;19(5):508-15.

4. Carter LE, Cook DP, Vanderhyden BC. Chapter 33 - Phenotypic Plasticity and the Orig- 
ins and Progression of Ovarian Cancer. In: Leung PCK, Adashi EY, editors. The Ovary (Third Edition) [Internet]. Academic Press; 2019 [cited 2020 Oct 2]. p. 529-45. Available from: http://www.sciencedirect.com/science/article/pii/B9780128132098000339

5. Levine RA, Hagége AA, Judge DP, Padala M, Dal-Bianco JP, Aikawa E, et al. Mitral valve diseasemorphology and mechanisms. Nat Rev Cardiol. 2015 Dec;12(12):689-710.

6. Kovacic JC, Dimmeler S, Harvey RP, Finkel T, Aikawa E, Krenning G, et al. Endothelial to Mesenchymal Transition in Cardiovascular Disease: JACC State-of-the-Art Review. J Am Coll Cardiol. 2019 22;73(2):190209.

7. Chen P-Y, Qin L, Tellides G, Simons M. Fibroblast growth factor receptor 1 is a key inhibitor of TGF $\beta$ signaling in the endothelium. Sci Signal. 2014 Sep 23;7(344):ra90.

8. Paruchuri S, Yang J-H, Aikawa E, Melero-Martin JM, Khan ZA, Loukogeorgakis S, et al. Human pulmonary valve progenitor cells exhibit endothelial/mesenchymal plasticity in response to vascular endothelial growth factor-A and transforming growth factor-beta2. Circ Res. 2006 Oct 13;99(8):861-9.

9. Liu AC, Joag VR, Gotlieb AI. The emerging role of valve interstitial cell phenotypes in regulating heart valve pathobiology. Am J Pathol. 2007 Nov;171(5):1407-18.

10. Fornes P, Heudes D, Fuzellier JF, Tixier D, Bruneval P, Carpentier A. Correlation between clinical and histologic patterns of degenerative mitral valve insufficiency: a histomorphometric study of 130 excised segments. Cardiovasc Pathol Off J Soc Cardiovasc Pathol. 1999 Apr;8(2):81-92.

11. Acker MA, Parides MK, Perrault LP, Moskowitz AJ, Gelijns AC, Voisine P, et al. Mitral-valve repair versus replacement for severe ischemic mitral regurgitation. N Engl J Med. 2014 Jan 2;370(1):23-32.

12. Topilsky Y. Mitral plasticity: possible target for intervention in patients with ischaemic mitral regurgitation? Eur Heart J - Cardiovasc Imaging. 2018 May 1;19(5):501-2.

13. Wylie-Sears J, Levine RA, Bischoff J. Losartan inhibits endothelial-to-mesenchymal transformation in mitral valve endothelial cells by blocking transforming growth factor- $\beta$-induced phosphorylation of ERK. Biochem Biophys Res Commun. 2014 Apr 18;446(4):870-5.

14. Man S, Sanchez Duffhues G, ten Dijke P, Baker D. The therapeutic potential of targeting the endothelialto-mesenchymal transition. Angiogenesis. 2019 Feb 1;22(1):3-13.

15. Sewell-Loftin MK, Chun YW, Khademhosseini A, Merryman WD. EMT-Inducing Biomaterials for Heart Valve Engineering: Taking Cues from Developmental Biology. J Cardiovasc Transl Res. 2011 Jul 13;4(5):658.

16. Calafiore AM, Totaro A, De Amicis V, Pelini P, Pinna G, Testa N, et al. Surgical mitral plasticity for chronic ischemic mitral regurgitation. J Card Surg. 2020 Apr;35(4):772-8. 